\title{
Grupo de educação em saúde para pessoas com esclerose lateral amiotrófica, seus familiares e cuidadores
}

\author{
Health education group for people with amyotrophic lateral sclerosis, their families \\ and caregivers
}

\section{Grupo de educación en salud para personas con esclerosis lateral amiotrófica, sus} familiares y cuidadores

Recebido: $23 / 04 / 2020$

Aprovado: 01/11/2020

Publicado: 07/03/2021

\author{
Crystian Moraes Silva Gomes ${ }^{1}$ \\ Ana Raquel Silva ${ }^{2}$ \\ Jonaina Fiorim Pereira de Oliveira ${ }^{3}$
}

Este é um relato de experiência, realizado no período de novembro de 2018 a dezembro de 2019, num Centro Especializado de Reabilitação de Vila Velha, Espirito Santo, com objetivo de descrever a experiência de constituição de um grupo multidisciplinar de educação em saúde, com pessoas diagnosticadas com Esclerose Lateral Amiotrófica, seus familiares e cuidadores. 0 grupo se intitulou "Café com Ela" $e$ houveram oito encontros com a participação de três a nove pacientes e quatro a 21 cuidadores ou familiares. As temáticas escolhidas se deram a partir das demandas dos participantes. 0 grupo foi caracterizado como homogêneo quanto às condições de saúde dos membros, e aberto com relação à entrada em cada encontro, sendo sempre conduzidos e construídos de forma multidisciplinar. A abordagem de grupo no contexto da reabilitação promoveu acesso a informação, maior conhecimento sobre a Esclerose Lateral Amiotrófica, apoio emocional através do compartilhamento de experiências, ampliação e construção de redes sociais e de suporte para o enfrentamento da doença.

Descritores: Esclerose Amiotrófica Lateral; Educação em saúde; Cuidadores; Reabilitação; Serviços de reabilitação.

This is an experience report, carried out from November of 2018 to December of 2019, in a Specialized Rehabilitation Center in Vila Velha, in the state of Espírito Santo. It aimed to describe the experience of setting up a multidisciplinary group of health education, with people diagnosed with Amyotrophic Lateral Sclerosis, their families and caregivers. The group called itself "Café com Ela" (Coffee with ALS) and there were eight meetings with the participation of three to nine patients and four to 21 caregivers or family members. The themes chosen were based on the demands of the participants. The group was characterized as homogeneous in regards to the health conditions of its members, and open in regards to the entry into each meeting, and it was always conducted and built in a multidisciplinary way. The group approach in the context of rehabilitation promoted access to information, greater knowledge about Amyotrophic Lateral Sclerosis, emotional support through the sharing of experiences, expansion and construction of social and support networks for coping with the disease.

Descriptors: Amyotrophic Lateral Sclerosis; Health education; Caregivers; Rehabilitation; Rehabilitation services.

Este es un informe de experiencia, realizado en el período de noviembre de 2018 a diciembre de 2019, en un Centro Especializado en Rehabilitación en Vila Velha, Estado de Espirito Santo, con el objetivo de describir la experiencia de la creación de un grupo multidisciplinario de educación en salud con personas diagnosticadas con Esclerosis Lateral Amiotrófica, sus familiares y cuidadores. El grupo se llamó "Café com Ela" (Café con Ela) y se celebraron ocho reuniones en las que participaron de tres a nueve pacientes y de cuatro a 21 cuidadores o familiares. Los temas elegidos se basaron en las demandas de los participantes. El grupo se caracterizó por ser homogéneo en cuanto a las condiciones de salud de los miembros, y abierto en cuanto a la entrada en cada reunión, siendo siempre conducido y construido de forma multidisciplinar. El enfoque grupal en el contexto de la rehabilitación promovió el acceso a la información, un mayor conocimiento sobre la Esclerosis Lateral Amiotrófica, el apoyo emocional a través del intercambio de experiencias, la ampliación y la construcción de redes sociales y de soporte para afrontar la enfermedad.

Descriptores: Esclerosis Amiotrófica Lateral; Educación en salud; Cuidadores; Rehabilitación; Servicios de rehabilitación.

1. Terapeuta Ocupacional. Especialista em Orientação e Mobilidade. Mestre em Psicologia. Doutorando em Psicologia pela Universidade Federal do Espirito Santo e atua no Centro de Reabilitação Física do Espírito Santo (CREFES), Vila Velha, ES, Brasil. 0RCID: 0000-0003-0675-0868 E-mail: crystian_salazar@hotmail.com

2. Terapeuta Ocupacional. Especialista em Terapia da Mão e Reabilitação Neurológica em Terapia Ocupacional. CREFES, Vila Velha, ES, Brasil. ORCID: 0000-0001-7399-2280E-mail: anaraquelquintas@gmail.com

3. Fisioterapeuta. Especialista em Reabilitação Cardíaca. Mestre e Doutora em Ciências Fisiológicas. CREFES, Vila Velha, ES, Brasil. ORCID: 0000-0002-6127-4596 E-mail: nanafiorim@hotmail.com 


\section{INTRODUÇÃO}

1 esclerose lateral amiotrófica (ELA) é uma doença progressiva e neurodegenerativa $\triangle$ associada à perda de neurônios motores superiores e inferiores, sendo caracterizada $\angle$ por fraqueza da musculatura estriada esquelética, com declínio progressivo da capacidade funcional, acompanhada de disartria, disfagia e falência da musculatura respiratória ${ }^{1-3}$.

Estudos recentes relatam que a progressão da ELA varia entre indivíduos, sendo a sobrevida média dos pacientes de 3 a 5 anos. No entanto, cerca de $10 \%$ dos casos apresentam uma forma de progressão mais lenta da doença e podem atingir uma sobrevida de mais de uma década $^{1-3}$. Assim, a severidade da doença e as incertezas quanto ao curso, o agravamento de incapacidades e a evolução da carga de cuidados são causa de grande estresse para os indivíduos afetados e suas famílias ${ }^{4}$.

A etiologia da ELA permanece desconhecida, os mecanismos fisiopatológicos parecem multifatoriais com evidência de uma interação complexa entre vias genética e molecular. Evidências sugerem vários mecanismos envolvidos, sendo as principais: mutação na enzima $\mathrm{Cu} / \mathrm{Zn}$ da Superóxido dismutase 1 (SOD1), que pode levar a toxicidade pelo aumento de superóxido; aumento do glutamato que leva a excitoxicidade com o acúmulo de cálcio intracelular; acúmulo de neurofilamentos; disfunção mitocondrial; ativação da micróglia e das células $\mathrm{T}$ e a presença de autoanticorpos ${ }^{5}$. Atualmente, o componente imunológico tem sido alvo de pesquisas no desenvolvimento da doença, e acredita-se que os fatores ambientais e uma predisposição genética sejam os responsáveis pela autoimunidade ${ }^{5,6}$.

O manejo clínico de pessoas com ELA é complexo e requer uma abordagem abrangente e multidisciplinar. As propostas gerais do cuidado são maximizar o desempenho funcional e melhorar a qualidade de vida. $\mathrm{O}$ foco do cuidado pode mudar à medida que a doença progride. Assim, entender a progressão da doença permite aos profissionais envolvidos no processo de reabilitação se antecipar às mudanças nas prioridades de atendimento e prevenir possíveis complicações ${ }^{1,3}$.

Pessoas com ELA apresentam necessidades multidisciplinares devido ao processo complexo e dinâmico da doença, beneficiando-se com intervenções de reabilitação individualizadas e/ou em grupo com o objetivo de otimizar a independência, a funcionalidade e a segurança, auxiliando a minimizar a carga sintomática e a maximizar a qualidade de vida 4 .

O Relatório Mundial das Deficiências ${ }^{7}$ (2012) descreve reabilitação como um conjunto de medidas que ajudam pessoas com deficiências ou prestes a adquirir deficiências a terem e manterem uma funcionalidade ideal na interação com seu ambiente. Desta forma, o processo de reabilitação envolve a identificação dos problemas e necessidades da pessoa, o relacionamento dos transtornos aos fatores relevantes do indivíduo e do ambiente, a definição de metas de reabilitação, planejamento e implantação de medidas e avaliação de seus efeitos. 0 documento ainda ressalta que educar as pessoas com deficiência é fundamental para o desenvolvimento de conhecimentos e habilidades para a autoajuda, a assistência, a gestão e a tomada de decisões.

A reabilitação do paciente com ELA exige da equipe multidisciplinar muita criatividade para melhorar e/ou manter a máxima funcionalidade possível, pois a doença pode evoluir de maneira rápida e diversificada. $\mathrm{Na}$ fase precoce são indicados exercícios físicos e uso de equipamentos adaptados; à medida que a doença progride são indicados medicamentos e recursos para controle da dor, espasticidade, cãibras, gerenciamento de secreções/sialorréia, fadiga, sono, capacidade respiratória e comunicação, além da intensificação do uso de tecnologia assistiva ${ }^{4}$.

A doença em fase avançada exige gerenciamento multimodal dos sintomas, planejamento de cuidados para o fim da vida e utilização oportuna de cuidados paliativos. É importante 
ressaltar que os aspectos emocionais e psicológicos, bem como a qualidade de vida, devem ser levados em consideração desde o momento do diagnóstico e durante todo o curso da doença ${ }^{4}$.

A educação pode ser compreendida como uma técnica de reabilitação, pois pessoas com deficiências e suas famílias e/ou cuidadores conseguem melhorar a saúde e a funcionalidade quando são esclarecidos, orientados e empoderados com a aquisição de conhecimento em saúde, tornando-se parceiros ativos no processo de reabilitação.

A Carta de Ottawa ${ }^{8}$ (1986) conceitua Promoção da Saúde como o processo de capacitação da comunidade para atuar na melhoria da qualidade de vida e saúde, incluindo uma maior participação no controle deste processo, ressaltando ainda que indivíduos e comunidades devem ter oportunidade de conhecer e controlar os fatores determinantes da sua saúde, sendo ambientes favoráveis, o acesso à informação, bem como oportunidades para fazer escolhas mais saudáveis, os principais elementos capacitantes.

A abordagem de grupo proporciona ao paciente o desenvolvimento de habilidades pessoais e possibilidades de enfrentamento da doença ${ }^{9}$. 0 desenvolvimento de um grupo de reabilitação com pessoas com Esclerose Lateral Amiotrófica e seus familiares promove experiências positivas sobre viver uma vida significativa e compartilhada, apesar das incapacidades graves, além de apoiar os relacionamentos familiares internos e a ampliação das redes sociais ${ }^{10}$.

0 trabalho com grupos é uma alternativa para as práticas assistenciais, estes favorecem o aprimoramento de todos os envolvidos, nos aspectos pessoais e profissionais, por meio da valorização dos diversos saberes e da possibilidade de intervir criativamente no processo de saúde-doença11.

Este trabalho tem por objetivo descrever a experiência de constituição de um grupo multidisciplinar de educação em saúde, com pessoas diagnosticadas com Esclerose Lateral Amiotrófica, seus familiares e cuidadores.

\section{MÉTODO}

Trata-se de um estudo descritivo do tipo relato de experiência, o qual apresenta, descreve e analisa a experiência da constituição de um grupo de educação em saúde, desenvolvido em um Centro Especializado em Reabilitação, com pessoas com diagnóstico de Esclerose Lateral Amiotrófica, seus familiares e cuidadores. A atividade foi desenvolvida entre o final de 2018 até 2019.

O Centro de Reabilitação Física do Estado do Espírito Santo (CREFES) é um Centro Especializado em Reabilitação II (CERII), habilitado nas modalidades motora e auditiva, localizado em Vila Velha - Espírito Santo. O CREFES é um ponto de atenção ambulatorial à pessoa com deficiência e oferece o serviço de prescrição, confecção e entrega de órteses, próteses e meios auxiliares de locomoção.

Os pacientes com ELA são atendidos pela Unidade de Trabalho Neurológico Adulto, que conta com equipe composta por médico fisiatra, neurologista e nutrólogo, terapeuta ocupacional, fisioterapeuta, psicólogo, fonoaudiólogo, assistente social e educador físico. Neste setor são atendidos predominantemente indivíduos pós-acidente encefálico cerebral; entretanto foi observado ao final de 2018 um aumento no número de paciente com o diagnóstico de Esclerose Lateral Amiotrófica.

Essa mudança no padrão dos atendidos evidenciou para a equipe multidisciplinar a necessidade de elaboração de novas estratégias de reabilitação, visto que apenas o atendimento ambulatorial não era capaz de suprir as dúvidas, incertezas e medos frente ao diagnóstico, tratamento e ao futuro expostos pelos pacientes e seus familiares. Durante os momentos de reunião de equipe e estudos de casos, foi observado um padrão de repetição das dúvidas dos pacientes, além do desejo de conhecer outras pessoas com a mesma condição de saúde. Foi 
sugerido pelos pacientes e familiares, e acordado entre a equipe o desenvolvimento de um grupo de educação em saúde, a fim de acolher as demandas dos pacientes com ELA, seus familiares e cuidadores.

Para implantação e efetivação do grupo os pacientes foram inicialmente convidados durante os atendimentos ambulatoriais. É importante ressaltar que o convite não foi estruturado de forma a garantir apenas a adesão à abordagem, mas vislumbrando o levantamento dos temas mais relevantes para os mesmos, e suas contribuições para o planejamento, execução e desenvolvimento da proposta, tendo processo ativo de tomada de decisão. A ELA é uma doença altamente incapacitante, por isso, durante a reabilitação, o respeito, o encorajamento e a autonomia devem ser priorizados com o intuito de promover maior motivação pessoal e construção de vínculos.

Um grupo de educação para pacientes com ELA, seus familiares e cuidadores busca estimular a pessoa a encontrar recursos para lidar com as questões do adoecer, da doença, da diminuição da funcionalidade e dos seus efeitos sobre sua vida. A partir de encontros iniciais entre alguns pacientes e equipe multidisciplinar, foi sugerido pelos primeiros o nome do grupo, intitulado "Café com ELA"; a escolha está associada à predileção dos pacientes pelo Café e da necessidade de enfrentamento da doença de forma leve e descontraída.

Inicialmente estruturado e pactuado de forma participativa às perspectivas do Grupo Café com ELA, foram eleitos os temas que se construíram como projetos específicos de intervenção durante os encontros. 0 grupo foi caracterizado como homogêneo quanto a condições de saúde dos participantes, e aberto com relação à entrada destes, dos familiares e cuidadores em cada encontro, sendo sempre conduzidos e construídos de forma multidisciplinar.

Apesar dos encontros e temas serem previamente estruturados, eles ocorrem de forma interativa, permitindo o envolvimento de todos os participantes, não sendo, portanto, apenas uma sessão formal de ensino, ressaltando sempre o direito que cada participante possui no exercício da fala, na exposição das suas opiniões, de seus pontos de vistas e inclusive do uso do silêncio.

Este estudo faz parte da pesquisa "Acompanhamento de Reabilitação do Paciente Neurológico no Centro de Reabilitação Física do Espírito Santo" aprovada pelo Comitê de Ética em Pesquisa do Centro de Ciências da Saúde da Universidade Federal do Espírito, recebendo o parecer de número 3.628.685 de 2019 (CAEE: 21020919.0.0000.5060). Todas as etapas da pesquisa seguiram as orientações da Resolução nº 466/2012 e Resolução nº 510/2016 do Conselho Nacional de Saúde.

\section{RESULTADOS}

O grupo Café com ELA foi desenvolvido entre novembro de 2018 e dezembro de 2019. Neste período foram desenvolvidos 8 encontros, com a participação de três a nove pacientes e quatro a 21 cuidadores ou familiares.

No encontro I, realizado em 14/12/2018 e intitulado "Conhecendo a Esclerose Lateral Amiotrófica", foram trabalhadas temáticas como: 0 que é ELA; Os mecanismos da doença; As principais doenças neurodegenerativas; A incidência e prevalência da ELA; Quais são as causas; Os fatores de risco; Quais são os sintomas da ELA; O que são doenças raras; Como é feito o diagnóstico da Esclerose Lateral Amiotrófica; Como é feito o tratamento e a reabilitação e quais são as possíveis complicações da doença.

Durante o encontro II, realizado em 20/03/2019, foram desenvolvidas temáticas referentes a "Reabilitação Respiratória" sendo trabalhado: 0 comprometimento dos músculos ventilatórios e a restrição pulmonar; Abordagem Fisioterapêutica na Esclerose Lateral Amiotrófica; Suporte Respiratório; Benefícios dos exercícios respiratórios para pacientes com ELA; Hipoventilação noturna; Avaliação da função respiratória (espirometria, gasometria, 
capacidade vital forçada, pico de fluxo de tosse, pressões respiratória, oximetria de pulso e Capnografia); Manejo da insuficiência respiratória em pacientes com ELA; Uso de suporte ventilatório invasivo e não invasivo; Ventilação não invasiva através de um dispositivo BiPAP; Técnicas de empilhamento de ar, por meio ressuscitador manual Ambu.

Os "Direitos das Pessoas com ELA" foram trabalhados no encontro III, realizado em 20/05/2019, que abordou: Os direitos específicos das pessoas com ELA e os análogos; Direitos sociais e fundamentais; Recursos de atendimento disponíveis em rede e suporte na comunidade; Portarias que se destinam ao atendimento da pessoa com ELA. Cadastro de sobrevida; Assistência domiciliar; Saque do FGTS; Serviço de transporte adaptado; Tratamento fora do Domicílio; Passe livre; Direitos previdenciários; Benefício de Prestação Continuada.

No IV encontro, desenvolvido em 30/05/2019, foi abordado o tema "A Mobilização de pessoas com ELA", discorrendo sobre: Melhoria da qualidade de vida dos pacientes, familiares e cuidadores; Articulação entre pacientes, familiares, cuidadores e profissionais da área da saúde para divulgação da ELA; Associações e entidades que trabalham pela garantia de direitos das pessoas com ELA, como Associação Brasileira de Esclerose Lateral Amiotrófica (AbrELA) e Associação Pró-Cura da Esclerose Lateral Amiotrófica.

Ainda no encontro IV, parte dos pacientes divulgaram a caminhada anual de conscientização da ELA desenvolvida pela AbrELA em São Paulo e demonstraram interesse em realizar um movimento semelhante no ES, devido a falta de informação sobre a doença e escassas iniciativas de divulgação; entretanto, pelo número reduzido de participantes, o pouco tempo para divulgação e pelas condições clínico funcionais de parte dos pacientes, foi descartada a hipótese de realização da caminhada.

Os pacientes e seus familiares então sugeriram uma atividade de panfletagem para divulgação de informações sobre a doença. Acordado o interesse e a importância do grupo para construção da atividade, pacientes, familiares e profissionais engajaram-se para efetivação da proposta, que foi considerada o $\mathrm{V}$ encontro do Café com ELA, intitulado "Vivendo com ELA aprendendo com eles", concretizada em 16/06/2019 através de uma Ação Educativa na Praia da Costa em Vila Velha-ES.

A escolha do local foi definida pela proximidade ao CREFES e por contar com estrutura acessível para pessoas com deficiência física, em virtude de um programa da Prefeitura para promoção de atividades de lazer adaptado. Através da mobilização e contato dos próprios pacientes foram disponibilizados banners e balões pela AbrELA, e panfletos e cartilhas de orientações pela Pró-Cura da ELA, a ação ainda contou com o apoio e participação da Associação Capixaba de Pessoas com Doenças Raras e doação dos próprios pacientes e familiares para aquisição de uma tenda. 
Figura 1 - Ação educativa desenvolvida no V encontro do Café com ELA. Vila Velha/ES, 2019.
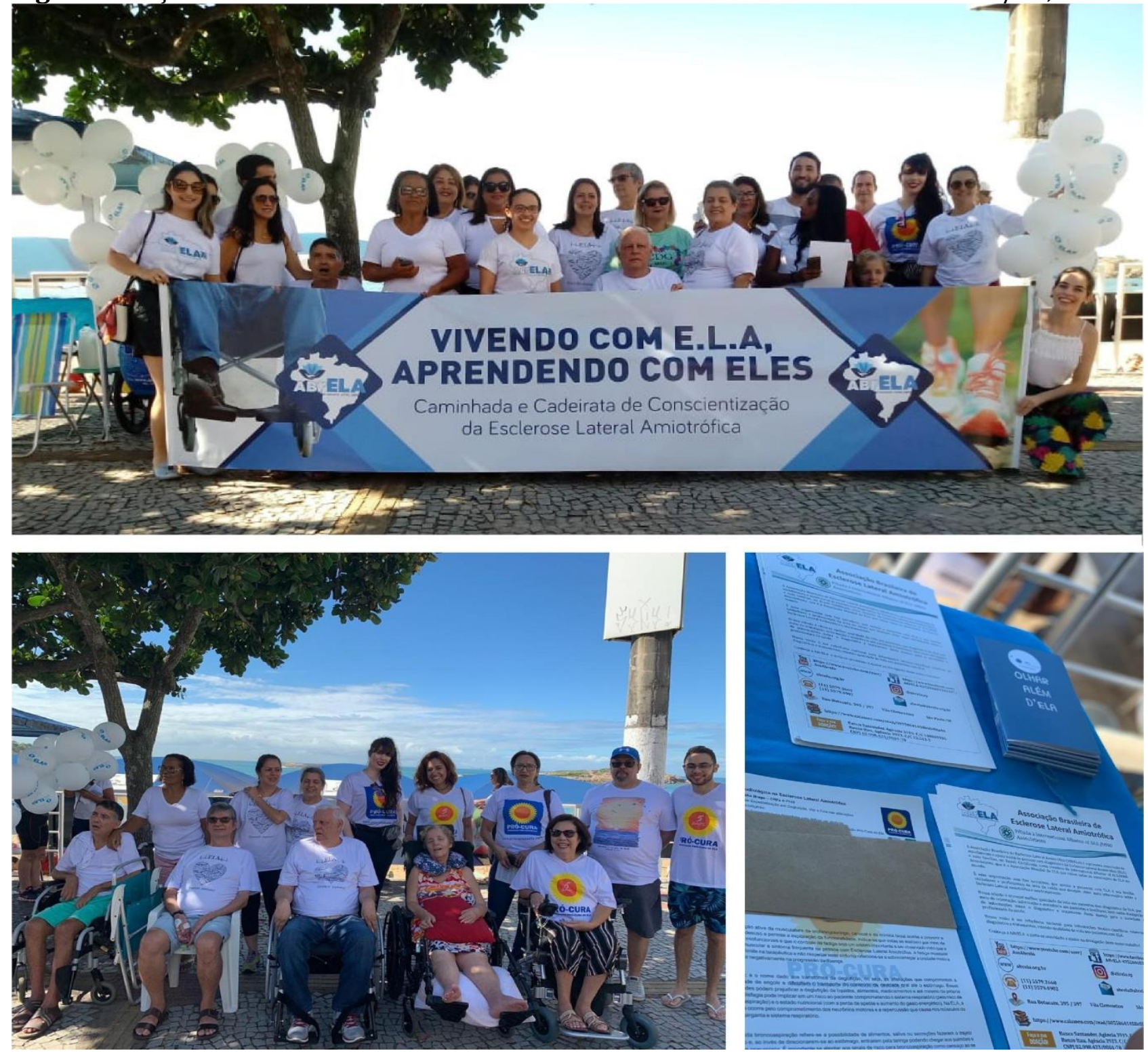

O Encontro VI nomeado "Cuidados com a Alimentação e Deglutição" 10/07/2019 discorreu sobre: Alterações da fonação e deglutição na Esclerose Lateral Amiotrófica; Disfagia na ELA; Disartria; Disfonia; Atuação do Fonoaudiólogo; Procedimentos compensatórios adquiridos; Exercícios miofuncionais e do aprendizado e técnicas que estimulam a propriocepção oral, alterações posturais e manobras de deglutição; A Intervenção Nutricional na Esclerose Lateral Amiotrófica; Mudanças nas características da alimentação (viscosidade, temperatura, sabor), modificações no volume e no ritmo da apresentação, e manobras intraorais; Aconselhamento Alimentar no Controle da Disfagia; Suporte Nutricional Entérico.

O "Uso de Recursos de Tecnologia Assistiva na ELA" foi tema do VII encontro em 04/09/2019, sendo discutido: 0 Uso de Tecnologia Assistiva para pacientes com Esclerose Lateral Amiotrófica; A prescrição adequada; 0 papel do terapeuta ocupacional da avaliação, prescrição, orientação sobre o uso e treino dos dispositivos; Confecção de órteses de membros superiores e Inferiores; Adequação postural em cadeira de rodas; Recursos assistivos de baixo e alto custo financeiro; Recursos Assistivos disponibilizados via Sistema Único de Saúde; Financiamentos existentes para aquisição de recursos de alto custo. 
O "Cuidado de quem Cuida" foi trabalhado no VIII encontro em 27/11/2019, abordando as temáticas: Sobrecarga do cuidador e redes de suporte social; Apoio social na experiência do familiar cuidador; Estresse emocional do cuidador; Cuidar de si e do outro; As fases do cuidado; Principais dificuldades encontradas nas tarefas do cuidar; Os sentimentos implicados no cuidado; Grupos de apoio; Convivência familiar e a importância do lazer.

Figura 2. Atividades desenvolvidas durante os encontros do Café com ELA. Vila Velha/ES, 2019.
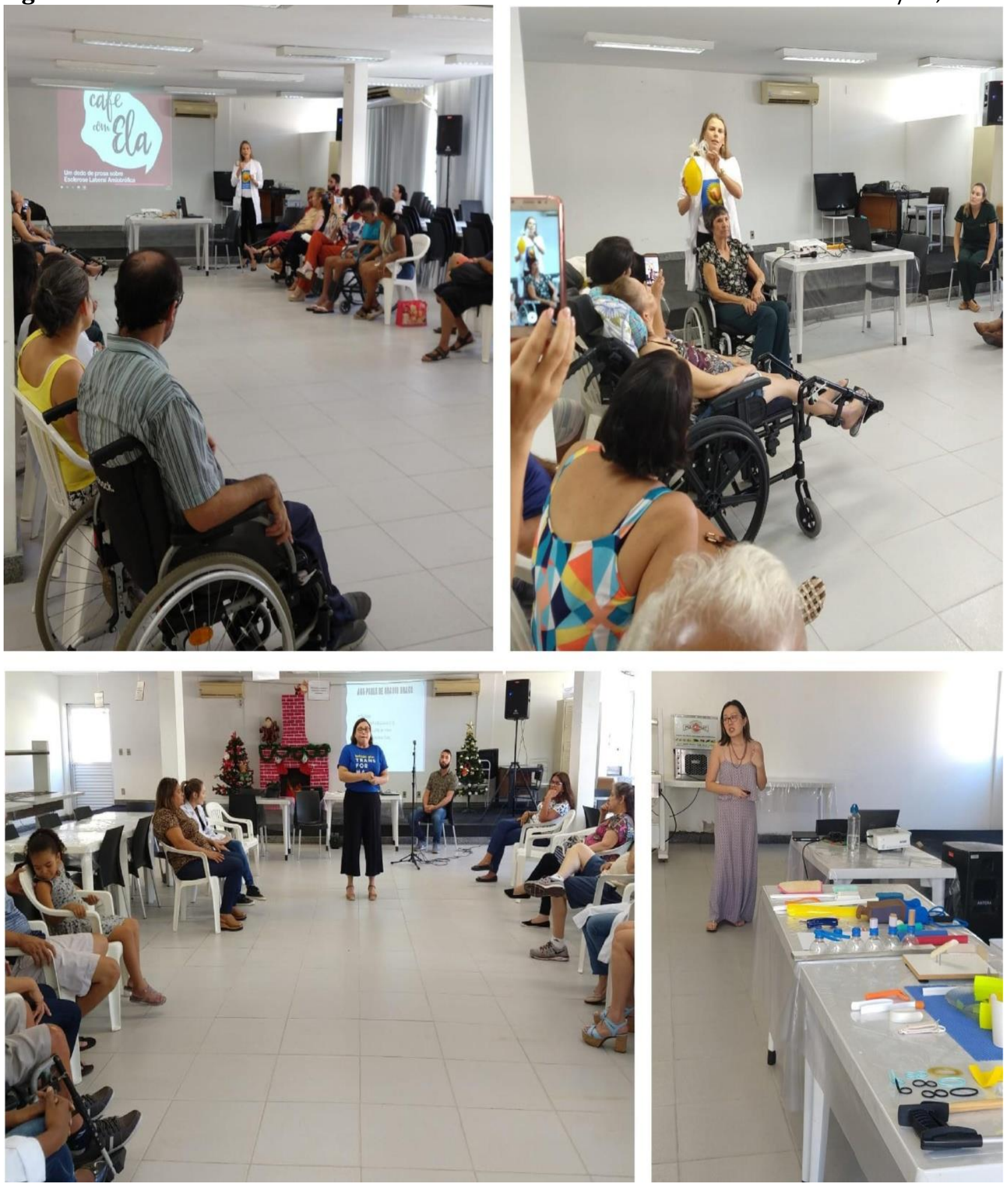

As duas primeiras imagens na parte superior foram realizadas no encontro II sobre "Reabilitação Respiratória”. As imagens da parte inferior foram realizadas no encontro VIII sobre "Cuidado de quem Cuida" e no encontro VII com o tema "Uso de Recursos de Tecnologia Assistiva na ELA". 
O número de participantes em cada encontro do Café com ELA pode ser visualizado por meio da Tabela 1.

Tabela 1. Participantes por edição do Café com ELA. Vila Velha/ES, 2019.

\begin{tabular}{ccccc}
\hline & & \multicolumn{2}{c}{ Participantes } & \\
Encontros & Pacientes & Familiares e Cuidadores & Profissionais & Total \\
\hline I & 5 & 5 & 8 & 18 \\
II & 9 & 21 & 11 & 41 \\
III & 4 & 4 & 8 & 16 \\
IV & 4 & 4 & 8 & 16 \\
V & 8 & 16 & 12 & 36 \\
VI & 9 & 10 & 9 & 28 \\
VII & 4 & 7 & 9 & 20 \\
VIII & 3 & 5 & 7 & 16 \\
\hline
\end{tabular}

\section{DISCUSSÃO}

Pessoas com ELA, seus familiares e cuidadores devem ser informados sobre a doença e seu curso. Observou-se para a maioria dos pacientes e seus familiares que o desconhecimento sobre a doença era mais difícil de lidar do que as informações sobre o que esperar à medida que a doença se agravasse. 0 conhecimento sobre os aspectos clínicos da doença, a redução da funcionalidade e o que pode acontecer no futuro facilitam a construção, adoção de medidas de apoio e a busca por assistência adequada em cada fase da doença. Necessidades de reabilitação não satisfeitas podem retardar a reabilitação, limitar atividades, restringir a participação, causar deterioração da saúde, aumentar a dependência pela assistência de outrem, e piorar a qualidade de vida 7 .

Os encontros do Café com ELA forneceram informações, dicas e ferramentas de enfrentamento úteis para a promoção do cuidado, estimulando a discussão entre pacientes, os membros de sua família e com os profissionais de saúde. A abordagem de grupo no contexto da reabilitação desempenha um papel importante para pessoas com ELA e seus familiares, fornecendo apoio social e emocional através do compartilhamento de experiências.

Um grupo pode ajudar pessoas durante períodos de ajustamento a mudanças, no tratamento de crises ou ainda na manutenção ou adaptação a novas situações, seu potencial emerge da possibilidade de pessoas com situações semelhantes compartilharem experiências comuns ${ }^{11}$. Através dos encontros, os participantes puderam aprender com indivíduos familiarizados com a situação enfrentada, compartilhar ideias com quem têm os mesmos desafios, e aprender como pessoas com ELA em fases avançadas estão lidando com problemas que poderão enfrentar no futuro.

Familiares e cuidadores de pessoas com ELA experimentam uma série de reações emocionais nesse contexto, sendo relatados sentimentos de injustiça por estar saudável enquanto um familiar enfrenta os desafios impostos pela doença. Alterações emocionais devido ao aumento das responsabilidades diárias impostas pelo acúmulo do papel de cuidador, além de sentimentos de culpabilidade por não saber como lidar com a doença do familiar, ou por reagir com pensamentos e atitudes negativas. Foram identificados durante os encontros déficits por parte dos pacientes e familiares no conhecimento, entendimento e manejo da doença.

A compreensão das necessidades educacionais dos pacientes permite aos profissionais de saúde elaborar programas em formato aceitável para os pacientes e seus familiares. Os participantes relataram melhor entendimento da doença, dos níveis de incapacidades decorrente do progresso da doença e na maneira de lidar com a situação. Mediante a aquisição de conhecimento sobre a ELA, da diversidade de sintomas e influência na redução da funcionalidade, os familiares expressaram melhora na compreensão dos comportamentos e 
capacidades das pessoas com ELA, e como lidar, compartilhar experiências e apoiar-se mutuamente na progressão da doença e das incapacidades ${ }^{10}$.

Estudo recente demonstrou que a autopercepção da deterioração da inteligibilidade da fala e do comprometimento respiratório estão diretamente relacionados com declínio da capacidade funcional em pacientes com diagnóstico de ELA ${ }^{1}$. A disartria grave, disfagia, ortopneia e dispneia tem grande impacto na qualidade de vida desses pacientes. Neste sentido, a preservação da autonomia e da capacidade de comunicação deve ser promovida pela equipe de reabilitação1. 0 número expressivo de participantes (pacientes e cuidadores) nos Encontros II e VI sugerem a carência de informações sobre as temáticas, o impacto do declínio funcional na qualidade de vida do paciente e na sobrecarga do cuidador. 0 grupo potencializou as trocas dialogadas, o compartilhamento de experiências e melhora nas adaptações de comunicação, suporte respiratório e aconselhamento alimentar ao modo de vida individual e coletivo.

Ao decorrer dos encontros, os participantes ficaram cada vez mais interessados nos temas abordados e em conhecer uns aos outros, expressando segurança e confiança na presença dos outros participantes do grupo. Sentimentos de solidão, desespero, perda e frustração por não ter em quem confiar, além de percepção de incompreensão da situação vivenciada, foram queixas dos familiares de pessoas com ELA antes da participação em grupos de educação e apoio ${ }^{10}$. A ampliação e construção de redes sociais e de apoio ficaram mais fortes ao longo do programa, alterando a dinâmica de grupo ao longo do tempo. Tal experiência proporcionou a ampliação da rede de apoio e a comunicação entre os participantes para além do grupo, por meio de troca de contatos, da criação de um grupo virtual com familiares e cuidadores, e da mobilização para divulgação de informações sobre a doença.

Os principais obstáculos para a participação de familiares de pessoas com ELA em um grupo de reabilitação são o medo de encontrar outras pessoas com ELA, a progressão da doença do familiar e problemas de acessibilidade e transporte ${ }^{10}$. A Tabela 1 demonstra uma variabilidade na adesão dos pacientes com diagnóstico de ELA e dos seus familiares e cuidadores ao longo dos encontros.

A justificativa da variabilidade na participação pode decorrer da negação do diagnóstico, do medo de conhecer a progressão da doença e seu declínio funcional, problemas de acessibilidade e transporte, precária rede de suporte no deslocamento até o serviço, e dificuldades socioeconômicas. Um componente positivo foi que, diante do impedimento da participação de alguns pacientes devido alto grau de dependência e progressão da doença, alguns familiares estiveram presentes nos encontros, potencializando sua capacidade para lidar com situações inerentes ao declínio funcional e auxiliando outros participantes do grupo a adquirirem maior confiança e alívio emocional.

Outro aspecto verificado pela equipe multidisciplinar que impactou a adesão dos participantes foram três óbitos de pacientes que ocorreram ao longo dos encontros. 0 primeiro óbito ocorreu antes do Encontro II, onde os vínculos entre os participantes não estavam bem consolidados. No entanto, os outros dois óbitos ocorreram antes do Encontro VII quando os vínculos entre os participantes já estava mais seguro, impactando emocionalmente inclusive os profissionais envolvidos. Além disso, soma-se a esses aspectos o temor à morte.

Um dos temas solicitados por familiares e cuidadores foram cuidados paliativos e terminalidade. A abordagem em cuidados paliativos no acompanhamento da pessoa com ELA visa melhorar a qualidade de vida do paciente e do familiar/cuidador diante de uma doença incurável e potencialmente fatal, aliviando a dor e outros sintomas angustiantes, afirmando a vida e considerando a morte como uma condição normal do processo de existência, integrando aspectos psicológicos e espirituais, e ofertando um sistema de apoio para ajudar famílias e cuidadores a lidar com os desafios antes e o depois da morte ${ }^{12}$. Apesar da extrema relevância do tema a equipe multidisciplinar avaliou não ser o momento mais oportuno para a discussão 
do tema, devido a precocidade da constituição do grupo e a falta de amadurecimento e elaboração da possibilidade de morte entre os participantes.

Devido à natureza progressiva da ELA, deve-se adotar um olhar criativo sobre o paradigma da reabilitação, o que requer o envolvimento de terapeutas familiarizados $\mathrm{e}$ experientes com as complexidades da doença ${ }^{13}$. O Café com ELA possibilitou a troca de conhecimento e experiências entre profissionais de saúde com diferentes titulações e saberes, oportunizando a comunicação e o trabalho em equipe, a uniformização de conceitos entre os profissionais, constituindo-se como um espaço de educação permanente em saúde. Além de favorecer a construção de alianças terapêuticas com os pacientes e seus familiares, para o atendimento de necessidades imediatas e futuras.

\section{CONCLUSÃO}

Os serviços de reabilitação precisam ser constantemente aprimorados para se adequar às necessidades das pessoas com Esclerose Lateral Amiotrófica. Frequentemente o acompanhamento ambulatorial não é capaz de acolher todas as demandas dos pacientes e seus familiares, levando a um baixo nível de conhecimento sobre a doença e interpretações equivocadas sobre a diminuição da funcionalidade e dos seus efeitos sobre sua vida.

A abordagem em grupo no contexto de reabilitação e a participação no programa, forneceram um ambiente de aprendizado para as pessoas com ELA e seus familiares, promovendo o apoio emocional através do compartilhamento de experiências, ampliação e construção de redes sociais e de suporte para o enfrentamento da doença.

Em relação às limitações encontradas, verificou as questões de planejamento e organização do fluxo de atendimento, uma vez que era nova a abordagem de educação em saúde como parte integrante das ações permanentes do Ambulatório de Tratamento Neurológico Adulto do CREFES. Como perspectiva futura sugere-se mensurar o nível de conhecimento dos participantes através de instrumentos de avaliação, possibilitando a mensuração quantitativa de mudanças ao longo da participação no grupo.

Por sua vez, espera-se que a experiência aqui apresentada estimule a prática da educação em saúde no contexto da reabilitação, em outras localidades e realidades, em especial, a sua aplicabilidade no tratamento de pessoas com Esclerose Lateral Amiotrófica.

\section{REFERÊNCIAS}

1. Londral A, Pinto A, Pinto S, Azevedo L, Carvalho M. Quality of life in amyotrophic lateral sclerosis patients and caregivers: impact of assistive communication from early stages. Muscle Nerve [Internet]. 2015 [citado em 21 abr 2020]; 52(6):933-41. Disponível em: https://www.ncbi.nlm.nih.gov/pubmed/258086. DOI: 10.1002/mus.24659

2. Hobson EV, McDermott CJ. Supportive and symptomatic management of amyotrophic lateral sclerosis. Nat Rev Neurol. [Internet]. 2016 [citado em 21 abr 2020]; 12(9):526. Disponível em: https://www.ncbi.nlm.nih.gov/pubmed/27514291. DOI: 10.1038/nrneurol.2016.111

3. Dal Bello-Haas V. Physical therapy for individuals with amyotrophic lateral sclerosis: current insights. Degener Neurol Neuromuscul Dis. [Internet]. 2018 [citado em 21 abr 2020]; 8:45. Disponível em: https://www.ncbi.nlm.nih.gov/pmc/articles/PMC6065609. DOI: 10.2147/DNND.S146949

4. Paganoni S, Karam C, Joyce N, Bedlack R, Carter GT. Comprehensive rehabilitative care across the spectrum of amyotrophic lateral sclerosis. NeuroRehabilitation. [Internet]. 2015 [citado em 21 abr 2020]; 37(1):53-68. Disponível em: https://content.iospress.com/articles/neurorehabilitation/nre1240. DOI: 10.3233/NRE-151240

5. Siqueira MK, Pires L. Processo epigenéticos envolvidos na fisiopatologia da esclerose múltipla com ênfase na função dos miRNAs. Semina Ciênc Biol Saúde [Internet]. 2016 [citado em 21 abr 2020]; $37(2): 125-36$. 
http://www.uel.br/revistas/uel/index.php/seminabio/article/view/24511. DOI: 10.5433/16790367.2016v37n2p125

6. Morgan S, Orrell RW. Pathogenesis of amyotrophic lateral sclerosis. Br Med Bull. [Internet]. 2016 [citado em 21 abr 2020]; 119(1):87-97. Disponível em: https://www.ncbi.nlm.nih.gov/pubmed/27450455. DOI: 10.1093/bmb/ldw026

7. World Health Organizaton, World Bank. Relatório mundial sobre a deficiência [Internet]. Trad Lexicus Serv Linguíst. São Paulo: Governo do Estado (São Paulo), Secretaria dos Direitos da Pessoa com Deficiência; 2012 [citado em 21 jan 2021]. 99p. Disponível em: https://portaldeboaspraticas.iff.fiocruz.br/wp-content/uploads/2020/09/9788564047020_por.pdf 8. World Health Organizaton. The Ottawa Charter for Health Promotion. In: The 1st International Conference on Health Promotion; 1986; Ottawa, CA [Internet]. Geneva: WHO; Health promotion; [2012] [citado em 21 jan 2021]. Disponível em: https://www.who.int/teams/health-promotion/enhancedwellbeing/first-global-conference

9. Gomes CMS, Coutinho GC, Miyamoto ST. Efeitos do programa de educação em pacientes com artrite reumatoide do Serviço de Reumatologia do Hospital Universitário Cassiano Antônio de Moraes (HUCAM)-projeto piloto. Rev Ter Ocup. [Internet]. 2014 [citado em 21 abr 2020]; 24(3):250-8. Disponível em: http://www.revistas.usp.br/rto/article/view/58467. DOI: $0.11606 /$ issn.22386149.v24i3p250-258

10. Madsen LS, Jeppesen J, Handberg C. "Understanding my ALS”. Experiences and reflections of persons with amyotrophic lateral sclerosis and relatives on participation in peer group rehabilitation. Disabil Rehabil. [Internet]. 2019 [citado em 21 abr 2020]; 41(12):1410-8. Disponível em: https://www.ncbi.nlm.nih.gov/pubmed/29373921. DOI: 10.1080/09638288.2018.1429499

11. Dias V, Silveira D, Witt R. Educação em saúde: o trabalho de grupos em atenção primária. Rev APS [Internet]. 2009 [citado em 21 abr 2020]; 12(2):221-7. Disponível em: https://periodicos.ufjf.br/index.php/aps/article/view/14261

12. Barawid E, Covarrubias N, Tribuzio B, Liao S. The benefits of rehabilitation for palliative care patients. Am J Hosp Palliat Care [Internet]. 2015 [citado em 21 abr 2020]; 32(1):34-43. Disponível em: https://www.ncbi.nlm.nih.gov/pubmed/24301083. DOI: 10.1177/1049909113514474

13. Majmudar S, Wu J, Paganoni S. Rehabilitation in amyotrophic lateral sclerosis: why it matters. Muscle Nerve [Internet]. 2014 [citado em 21 abr 2020]; 50(1):4-13. Disponível em: https://www.ncbi.nlm.nih.gov/pmc/articles/PMC4433000/. DOI: 10.1002/mus.24202

\section{Editora Associada: Estefânia Maria Soares Pereira}

\section{CONTRIBUIÇõES}

Crystian Moraes Silva Gomes e Jonaina Fiorim Pereira de Oliveira participaram da concepção do estudo, coleta e análise e redação. Ana Raquel Silva contribuiu na concepção do estudo e revisão.

\section{Como citar este artigo (Vancouver)}

Gomes CMS, Silva AR, Oliveira JFP. Grupo de educação em saúde para pessoas com Esclerose Lateral Amiotrófica, seus familiares e cuidadores. REFACS [Internet]. 2021 [citado em inserir dia, mês e ano de acesso]; 9(Supl. 1):323-333. Disponível em: inserir link de acesso. DOI: inserir link do DOI

\section{Como citar este artigo (ABNT)}

GOMES, C. M. S.; SILVA, A. R.; OLIVEIRA, J. F. P. de. Grupo de educação em saúde para pessoas com Esclerose Lateral Amiotrófica, seus familiares e cuidadores. REFACS, Uberaba, MG, v. 9, p. 323-333, 2021. Supl. 1. D0I: inserir link do DOI. Disponível em: inserir link de acesso. Acesso em: inserir dia, mês e ano de acesso.

\section{Como citar este artigo (APA)}

Gomes, C.M.S., Silva, A.R., \& Oliveira, J.F.P. (2021). Grupo de educação em saúde para pessoas com Esclerose Lateral Amiotrófica, seus familiares e cuidadores. REFACS, 9(Supl. 1), 323-333. Recuperado em inserir dia, mês e ano de acesso de inserir link de acesso. DOI: inserir link do DOI. 